\title{
A Follow-Up Study of Painful Diabetic Neuropathy: Physical and Psychological Aspects
}

\author{
Yoshiatsu Takahashi and Yukimasa Hirata \\ Diabetes Center, Tokyo Women's Medical College, \\ Shinjuku-ku, Tokyo 162
}

\begin{abstract}
Takahashi, Y. and Hirata, Y. A Follow-Up Study of Painful Diabetic Neuropathy: Physical and Psychological Aspects. Tohoku J. exp. Med., 1983, 141(4), 463-471— Twenty-one patients with painful diabetic neuropathy were followed up for about 18 months by means of physical and psychological tests. Patients with painful diabetic neuropathy had significantly more neurotic, anxious and depressive traits than those with non-painful diabetic neuropathy. During the course of the study, 17 patients out of 21 were found to be relieved of their neuropathic symptoms with improved blood glucose control and medications. However, despite this improvement, diabetic retinopathy and nephropathy of the patients deteriorated. Patients with improved neuropathy had gained body weight, with a concomitant relief of depression, nervous tendency and anxiety. However, lack of extroversion was not changed at all. Patients with painful diabetic neuropathy were significantly less extroversive than those with nonpainful diabetic neuropathy, even when their neuropathic symptoms might have improved. This study shows that although nervousness, anxiety and depressive tendencies may be secondary to the symptoms of painful diabetic neuropathy, lack of extroversion can be a primary characteristic trait which may easily induce painful diabetic neuropathy. —— painful diabetic neuropathy; psychological tests ; depression ; extroversion
\end{abstract}

In our daily clinical observations, we often find that patients with painful diabetic neuropathy (PDN) are resistant to medical therapy using vitamines, analgesics and tranquilizers, even if their blood glucose level is well-controlled. By using psychological tests, we have been able to identify the characteristic features of the patients with PDN ; i.e. most of them are middle aged, thin men with neurotic, anxious and depressive traits in comparison with those with nonpainful diabetic neuropathy (NPDN). However, it is uncertain whether these characteristic features are primary or secondary to the painful symptoms. This follow-up study was therefore undertaken to investigate the causes of the psychological aspects of PDN.

Received for publication, May 12, 1983. 


\section{Subjects And Methods}

Twenty-one consecutive patients were referred to our hospital from 1979 to 1981 with a diagnosis of diabetic neuropathy with leg pain and/or numbness. They were treated mainly by vitamines $\left(\mathrm{B}_{6}, \mathrm{~B}_{12}\right)$ and carbamazepine concomitantly with the control of blood glucose. About 18 months later, 17 patients out of 21 showed improvement of their neuropathic symptoms in the extremities but the remaining 4 did not. These 17 patients with improved PDN (iPDN) were accepted for study within the age range of 31 to 68 years. They consisted of 5 women and 12 men and were injected with insulin except one who took an anti-hyperglycemic drug. The duration of diabetes mellitus was 3 to 15 years with a mean of 8.2 years. The duration of PDN was 2 to 24 months with a mean of 12.7 months.

Another group of subjects were 20 diabetics with non-painful diabetic neuropathy (NPDN), whose age, sex, duration of diabetes and $\mathrm{HbA}_{1}$ were all matched with those of the patients with PDN (Table 1). Control data for the questionnaires were obtained from the patients with NPDN.

All patients were subjected to 5 personality tests during their stay in the hospital (patients with PDN and NPDN) and at their homes (iPDN). They were told that the tests were personality tests and were part of a research program on patients with diabetic neuropathy.

In addition to the routine physical and standard neurological examinations, we measured $\mathrm{NCV}$ and $\mathrm{HbA}_{1}$ as an indication of blood glucose control during the last 6-week period. The normal value comprises $5.5 \sim 8.5 \%$ of the total hemoglobin.

\section{Cornell medical index (CMI)}

The CMI is Fukamachi's revised version indicating the grade of neurotic tendencies (Fukamachi 1959). The CMI is measured by counting the number of positive answers to 160 somatic and 51 mental questions. The grade of CMI is as follows: I is normal, II is almost normal, III is slightly neurotic and IV is neurotic.

\section{Manifest anxiety scale (MAS)}

This personality test, which was translated into Japanese from Taylor's personality scale of maifest anxiety (Taylor 1953), measures the manifest generalized trait anxiety (Taylor et al. 1968). The total number of questions is 50. A score over 23 indicates very high anxiety, over 18 is high anxiety and under 18 means normal. A lie score over 11 means less reliability of the test.

\section{Maudsley personality inventory (MPI)}

The Japanese version of MPI had its origin in the paper of Eysenck (1956). MPI measures two characteristic features of extroversion and nervousness. The coefficient of reliability of each scale is reasonably high in spite of the relative shortness of the test. The normal range of both scales is the same; from 19 to 29 , and a lie score over 20 means unreliable.

\section{Self-rating depression score (SDS)}

This test was developed by Zung et al. (1965) and consists of a 20-item scale for rating a variety of symptoms associated with depression. The normal score is under 40 and a score over 50 indicates severe depression (Ikemi 1974). The test of SDS is so simple and easy that it is often available for clinicians to diagnose and follow up depression.

Yatabe-Guilford test (YG-test)

The YG-test is one of the most useful and popular personality tests in Japan. It was modified from the Guilford-Martin Inventory by Yatabe et al. (Tsujioka 1965). The test 
TABLE 1. Patients with painful diabetic neuropathy (PDN) and nonpainful diabetic neuropathy (NPDN)

\begin{tabular}{lccc}
\hline & PDN & NPDN & $t$-test \\
\hline Number of cases & 17 & 20 & - \\
Age (years) & $44 \pm 9$ & $45 \pm 11$ & n.s. \\
Sex ratio (M/F) & $12 / 5$ & $13 / 7$ & n.s. \\
Duration of DM (years) & $8.6 \pm 3.6$ & $8.3 \pm 6.8$ & n.s. \\
Grade of obesity $(\%)$ & $78.9+9.7$ & $107.3 \pm 15$ & $p<0.005$ \\
$\mathrm{HbA}_{1}(\%)$ & $12.1 \pm 2.5$ & $11.7 \pm 2.3$ & n.s. \\
\hline
\end{tabular}

Mean \pm s.D. $\quad$ n.s. $=$ not significant.

TABLE 2. Clinical difference between PDN and the patients with improved PDN (iPDN)

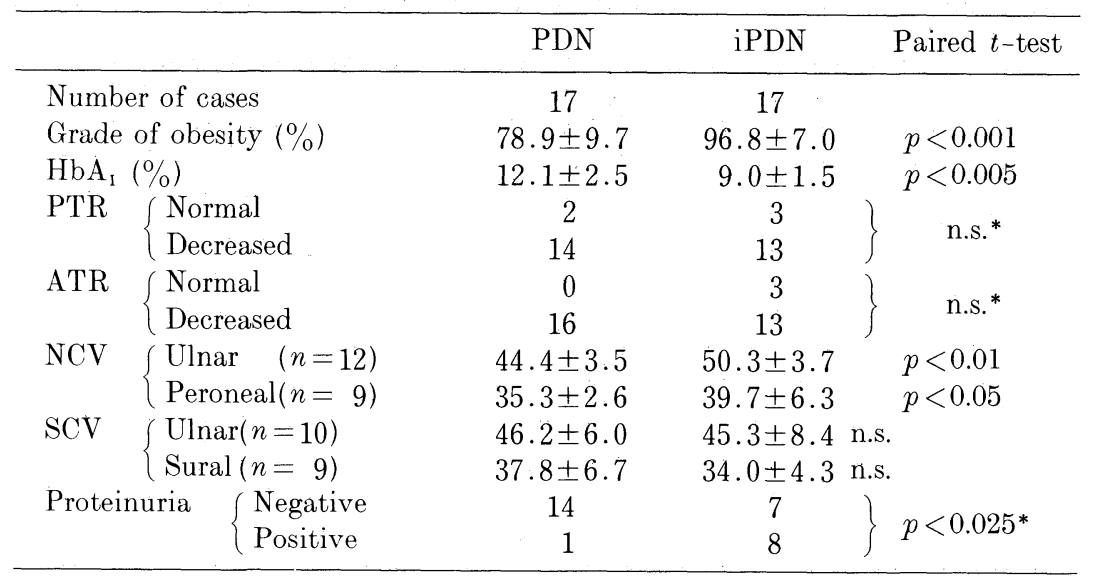

Mean \pm S.D. ${ }^{*} \chi^{2}$-test

comprises a 12-item scale of characteristic personalities and the normal range of each scale is about $10 \pm 3$.

\section{RESULTS}

Somatic and chemical findings

The grade of obesity of the patients with PDN was $78.9 \pm 9.7 \%$ (mean \pm s.D.) and that of the patients with iPDN was $96.8 \pm 7.0 \%$, the difference being significant by a paired $t$-test comparison $(p<0.001)$ (Table 2$)$. It was found that all patients with PDN who showed improvement in their neuropathic symptoms gained more than $10 \mathrm{~kg}$ in their body weight. However, those who showed no marked change in their symptoms gained less than $5 \mathrm{~kg}$.

$\mathrm{HbA}_{1}$ of the patients with PDN was $12.1 \pm 2.5 \%$ (mean \pm s.D.) and that of those with iPDN was $9.0 \pm 1.5 \%$, the difference being significant by a paired $t$-test comparison $(p<0.001)$ (Table 2$)$. $\mathrm{HbA}_{1}$ in all of the patients with PDN improved on treatment with insulin (16 patients) and drug (one patient).

The stages of diabetic retinopathy are described with Scott classification; i.e. 


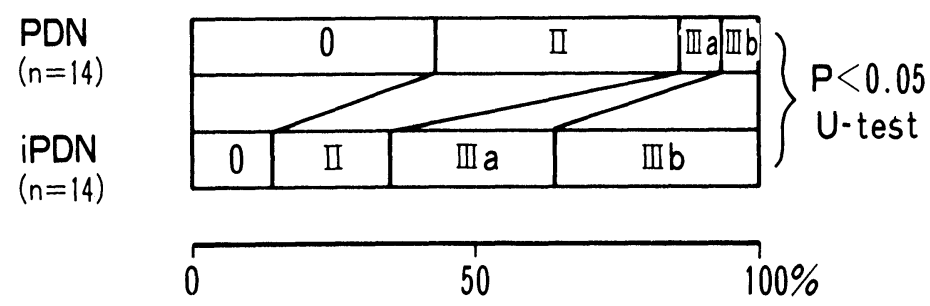

Fig. 1. Distribution of retinopathy in PDN and iPDN by Scott classification.

Scott 0 is normal, Scott Ia, II, IIIa indicate background retinopathy and Scott IIIb is proliferative retinopathy. As shown in Fig. 1, there was a significant difference in the distribution of retinopathy between PDN and IPDN according to a U-test comparison $(p<0.05)$. Two patients with PDN had taken photo-coagulation for treatment of retinopathy and one patient could not complete the examination of fundoscopy.

Proteinuria of patient's spot urine was checked by albustics as a screening test for diabetic nephropathy. The percentage of positive proteinuria was significantly increased in the patients with iPDN $(p<0.05)$ as shown in Table 2.

With regard to objective findings of diabetic neuropathy, we examined deep tendon reflexes and nerve conduction velosity. Both patellar tendon reflex (PTR) and axillary tendon reflex (ATR) slightly improved in the patients with iPDN. Ulnar and peroneal motor conduction velocity (MCV) improved significantly in the patients with iPDN $(p<0.05)$. However, ulnar and sural sensory nerve conduction velocity (SCV) did not (Table 2). Not all the subjects could not complete the tests of nerve conduction velocity and so the number of patients who were given the pairs of the tests were small.

\section{Results of psychological tests}

Fig. 2 shows the grade of CMI results on three groups. Concerning CMI, 8 of 16 patients with PDN showing neurotic tendencies improved, 7 were not changed and one became worse, the difference between PDN and iPDN being significant by

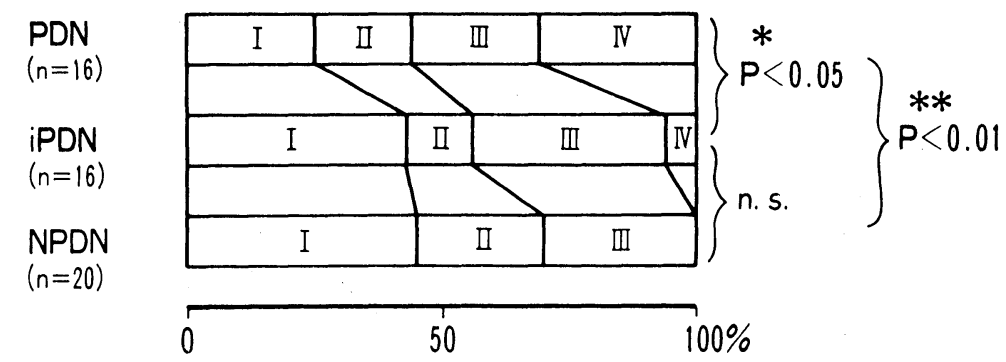

Fig. 2. The results of CMI in PDN, iPDM and NPDN. I is normal, II is almost normal, III is slightly neurotic and IV is neurotic. ${ }^{*}$ Paired $t$-test ${ }^{* *}$ Mann Whitney's U-test. 
CMI-somatic questions

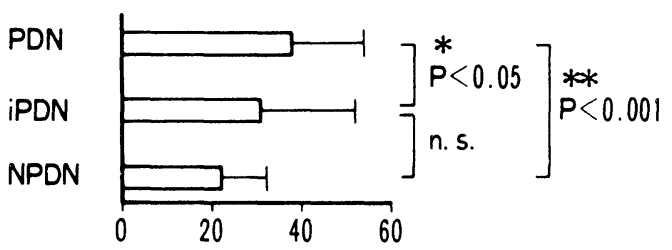

CMI-mental questions

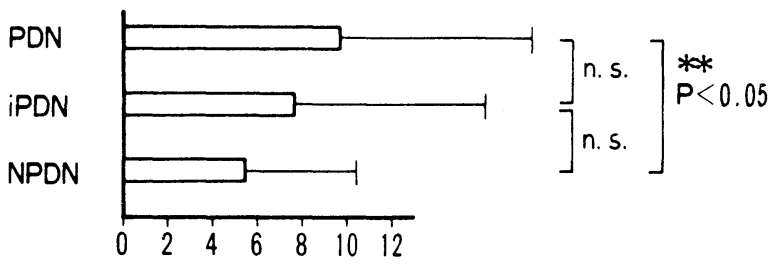

Fig. 3. CMI : the mean number of positive answers to somatic questions (upper) and mental questions (below). (mean \pm S.D.) ${ }^{*}$ Paired $t$-test $\quad{ }^{* *} t$-test.

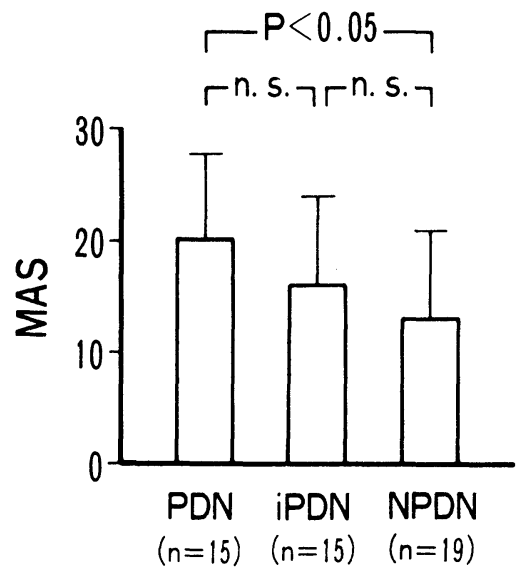

Fig. 4. MAS results in PDN, iPDN and NPDN (mean \pm s.D.).

a paired comparison $t$-test $(p<0.05)$. The distribution of the grade of CMI between PDN and NPDN was significantly different by a comparison U-test $(p<$ 0.01). However, the difference between iPDN and NPDN was not significant. One subject forgot to take a check of CMI.

Fig. 3 shows the number of positive answers on the CMI. The mean of positive answers to somatic questions in the patients with PDN was significantly different from those with iPDN $(p<0.05)$ and NPDN $(p<0.01)$. However, the difference between the patients with iPDN and those with NPDN was not significant by a comparison $t$-test. On the other hand, the mean of positive answers to mental questions in the patients with PDN was higher than that in those with NPDN. The difference between PDN and iPDN and the difference between 


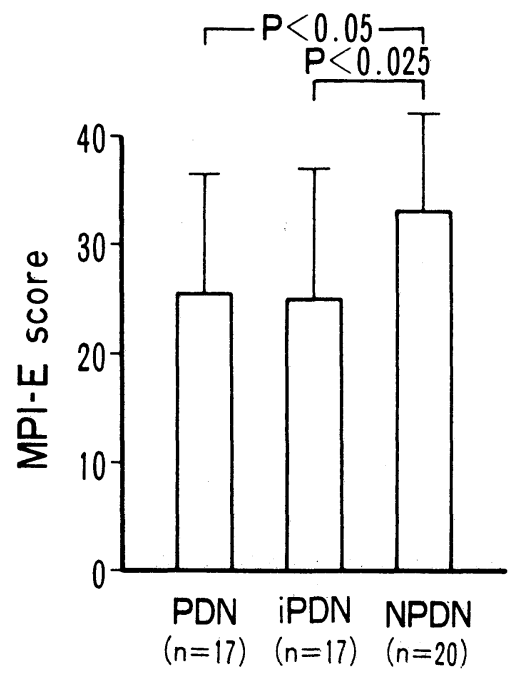

Fig. 5. MPI-E score in PDN, iPDN and NPDN (mean \pm s.D.).

between iPDN and NPDN were not significant because of large standard deviations.

The mean anxiety score for the patients with PDN was $20.5 \pm 7.9$ (mean \pm S.D.) ; that of the patients with iPDN was $16 \pm 7.9$ and that of the patients with NPDN was $12.9 \pm 8.1$, which was significantly different from PDN $(p<0.05)$ (Fig. 4). Two subjects were eliminated from this test because they did not complete the test.

The mean extroversion score of the patients with PDN was $26 \pm 12$ (mean \pm

TABle 3. The result of YG-test

\begin{tabular}{|c|c|c|c|}
\hline & $\operatorname{PDN}(n=17)$ & $\operatorname{iPDN}(n=17)$ & $\operatorname{NPDN}(n=20)$ \\
\hline Depression & $* 8.6 \pm 6.1^{\triangle \Delta}$ & * $5.7 \pm 5.0$ & $4.0 \pm 4.5^{\triangle \Delta}$ \\
\hline Cyclic tendency & $* * * 7.9 \pm 4.3$ & $* * * \quad 4.7 \pm 4.6$ & $5.9 \pm 4.8$ \\
\hline Inferiority & $5.1 \pm 5.5$ & $4.4 \pm 4.7$ & $4.1 \pm 3.9$ \\
\hline Nervous & $* * 7.7 \pm 5.3^{\triangle}$ & & \\
\hline Objectivity & $* 7.3 \pm 4.7$ & $* 5.1 \pm 5.0$ & $4.8 \pm 4.2$ \\
\hline Cooperative & $6.4 \pm 3.1$ & $6.1 \pm 3.8$ & $5.7 \pm 3.4$ \\
\hline Aggressive & $11.5 \pm 4.3$ & $10.9 \pm 4.8$ & $11.4 \pm 3.8$ \\
\hline General activity & $11.8 \pm 5.0$ & $12.0 \pm 5.2$ & $12.7 \pm 4.8$ \\
\hline Rhathymia & $9.5 \pm 4.0$ & $9.2 \pm 5.2$ & $11.2 \pm 3.9$ \\
\hline Thinking extroversion & $9.5 \pm 4.4^{\triangle \Delta}$ & $10.9 \pm 3.8$ & $13.0 \pm 4.4^{\triangle \triangle}$ \\
\hline Ascendance & $9.9 \pm 6.3$ & $10.6 \pm 5.4$ & $12.5 \pm 3.6$ \\
\hline Social extroversion & $12.2 \pm 4.7^{\triangle} \Delta$ & ${ }^{* *} 12.7 \pm 4.6$ & ${ }^{* *} 15.6 \pm 2.9^{\triangle} \triangle$ \\
\hline
\end{tabular}

Mean \pm S.D.

${ }_{\triangle}^{*} p<0.05, \quad{ }_{\triangle}^{* *} p<0.025, \quad * * * p<0.001$. 


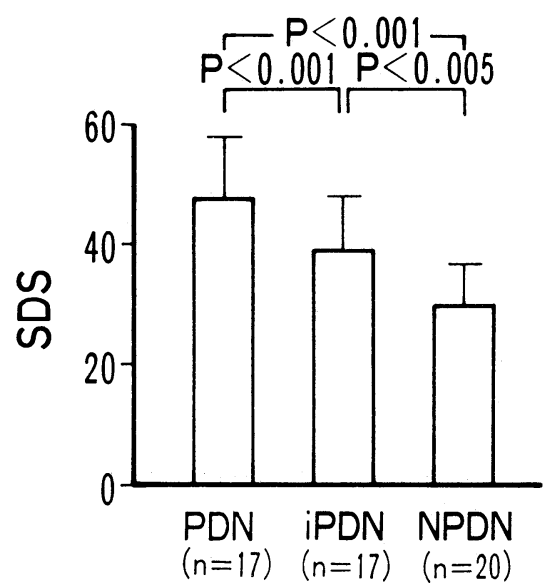

Fig. 6. SDS results in PDN, iPDN and NPDN (mean \pm s.D.).

S.D.) and that of iPDN was $25 \pm 12$, the difference being not significant. However, the score of the patients with NPDN was $33 \pm 9$, which was significantly different from that of both PDN $(p<0.05)$ and iPDN $(p<0.025)$ (Fig. 5). The score of nervousness and lie score were not different among the three groups.

The SDS of the patients with PDN was $48 \pm 10$ and that of iPDN was $39 \pm 9$, the difference being significant by a paired $t$-test comparison $(p<0.01)$. The score of the patients with NPDN was $30 \pm 7$, which was significantly different from the score of both PDN $(p<0.001)$ and $\operatorname{iPDN}(p<0.005)$ (Fig. 6$)$. In 15 out of 17 patients with PDN, the score of SDS improved during the course of study and two other patients varied within normal range.

Table 3 shows the results of YG-tests. The items such as depression, cyclic tendency, nervousness and objectivity were significantly different between PDN and iPDN ; however, those four items in iPDN were not different from those in NPDN. The items of thinking extroversion and social extroversion were not different between PDN and iPDN, and the scores of these characteristics in NPDN were significantly higher than in PDN and iPDN.

\section{Discussion}

"Painful diabetic neuropathy", as it is described in a current textbook (Ellenberg 1970), is a term typically diagnosed by most neurologists and internists when other possible causes of the pain are not apparent. Post-treatment neuropathy and apparently ischemic peripheral neuropathy are ruled out in this study. All of the subjects had severe painful symptoms or numbness in their extremities which were seldom responding to common medical therapy using vitamines, analgesics and tranquilizers. Therefore they were admitted to our hospital to determine the causes of the symptoms and to treat more effectively. In the course of study, PDN improved gradually by controlling blood glucose and increasing 
increasing body weight to a desirable level. With normalization of $\mathrm{HbA}_{1}$ and body weight, body weight, neurological findings also improved as the neuropathic symptoms diminished. On the other hand, aggravating retinopathy and nephropathy occurred despite the improvement of $\mathrm{HbA}_{1}$, which might be due to the period of poor control of blood glucose level before the start of treatment.

In this study, we have shown the psychological changes indicated by several personality tests in the course of treatment of PDN and have clarified the question as to whether the psychological aspects of PDN are primary or secondary to the symptoms themselves.

The CMI results showed that the patients with PDN had more nervous tendencies than those with NPDN and the nervous condition improved in the patients with iPDN during the treatment of PDN. As Okonogi et al. (1969) pointed out that the results of CMI could change in the course of the disease, we found significant difference of CMI results between PDN and iPDN. The number of positive answers to somatic questions significantly decreased whereas that of mental questions did not; therefore it would appear that the nervous tendencies in the patients with PDN might be secondary to the neuropathic symptoms.

Many studies have shown that the perception of pain is closely related to anxiety levels. Recently, two types of anxieties have been recognized; namely generalized trait anxiety and transient situation-induced anxiety (Evans 1974). Patients with PDN were more anxious than those with NPDN according to the result of MAS. However, the patients with iPDN had less anxiety than those with PDN and more anxiety than those with NPDN. This means that high anxiety in the patients with PDN may be a transient situation-induced anxiety, which is mainly produced by the painful symptoms.

It is generally thought that diabetics have an extroversive character (Matsunaga et al. 1965), as shown by the results of our MPI studies. The patients with both PDN and iPDN, however, were significantly more introversive than those with NPDN. The diabetics who are not so extroversive, therefore, might be subject to become PDN.

According to a controlled study (Greydanus and Hofmann 1979), diabetics, in particular those with poor control or long duration, are more depressive. Roger (1980) has reported recently that treatment with anti-depressant resulted in complete remission of PDN with concomitant relief of depression and return of depression test score. The test result of our study was in agreement with the conclusion of other workers ; i.e. the SDS did improve significantly when the pain disappeared. As SDS determines a depressive tendency at a certain time under a certain situation, depressive tendencies of the patients with PDN are thought to be due to the painful symptoms.

The YG-test is generally believed to indicate constant and native personality features, but a study has revealed that the test reflects the transient stability of emotion and adaptability in social life (Okonogi et al. 1969). The mean score of 
depression for the patients with PDN improved significantly, which was similar to the results of SDS. The item of nervousness also improved, as well as CMI results, in the patients with iPDN.

The items of thinking and social extroversion were not changed at all between the patients with PDN and iPDN, which were the same results as those of MPI. Therefore, lack of extroversion is considered to be a specific characteristic which predisposes the onset of PDN.

\section{Acknowledgment}

We particularly wish to thank Dr. M. Sugita, Kyushu University, for his kind assistance with this manuscript.

\section{References}

1) Ellenberg, M. (1970) Diabetic neuropathy. In: Diabetes Mellitas: Theory and Practice, edited by M. Ellenberg \& H. Rifkin, McGraw-Hill Book Co., Inc., New York, p. 824.

2) Evans, F.J. (1974) The placebo response in pain reduction. Advanc. Neurol., 4, 289-300.

3) Eysenk, H.J. (1956) The questionnaire measurement of neuroticism and extroversion. Riv. Psychol., 50, 113-140.

4) Fukamachi, K. (1959) The study on the Cornell Medical Index (1) The characteristic of complaints of neurotic patients showed on the CMI. Fukuoka med. J., 50, 29883000. (in Japanese with English abstract)

5) Greydanus, D.E. \& Hofmann, A.D. (1979) Psychological factors in diabetes mellitus. Amer. J. Dis. Child., 133, 1061-1066.

6) Ikami, Y. (1974) Modern Psychosomatic Medicine. lst ed., Ishiyaku Press, Tokyo, Chapter 10, p. 178. (Japanese)

7) Matsunaga, H., Kubo, A., Sato, H., Kawakami, S., Sato, M. \& Kikuchi, H. (1965) A psychosomatic study of diabetes mellitus. Jap. J. psychosomat. Med., 5, 15-21. (Japanese)

8) Okonogi, K., Yamamoto, K., Ueda, N. \& Yoshida, N. (1969) Clinical studies on the CMI in psychosomatic medicine II. Jap. J. psychosomat. Med., 9, 115-122. (Japanese)

9) Roger, W.T. (1980) Depression masquerading as diabetic neuropathy. $J$. Amer. med. Ass., 21, 1147-1150

10) Taylor, J.A. (1953) A personality scale of manifest anxiety. J. abnorm. soc. Psychol., 48, 285-290.

11) Taylor, J.A., Abe, M. \& Takaishi, N. (1968) The Guide book of MAS . Sankyobo, Kyoto. (Japanese)

12) Tsujioka, Y. (1965) The Guide Book of Using, Applying and Researching for YGPersonality Test. Japan Psychological Test Institute, Osaka. (Japanese)

13) Zung, W.W.K., Durham, N.C., Carolgn, B.R. \& Marvin, J.S. (1965) A self-rating depression scale in an outpatient clinic. Arch. gen. Psychiat., 13, 508-515. 